\title{
Hyperglycemia Risk Evaluation of Hydrocortisone Intermittent Boluses vs Continuous Infusion in Septic Shock: A Retrospective Study
}

\author{
Hassan Mitwally ${ }^{1}$, Mohamed O Saad ${ }^{2}$, Sara Mahmoud ${ }^{3}$, Adham Mohamed $^{4}$
}

\begin{abstract}
Introduction: Hydrocortisone showed to be effective in reducing the time until reversal of shock when added to standard therapy in managing septic shock. Hyperglycemia is one of the common adverse effects associated with corticosteroid treatment. However, the difference in hyperglycemia risk with different methods of hydrocortisone administration is not clear. The objective of this study was to evaluate the risk of hyperglycemia of intermittent hydrocortisone boluses vs continuous infusion in septic shock patients.

Materials and methods: This was a retrospective observational study. Data were collected from the electronic medical records of eligible patients admitted to intensive care units. All patients admitted with septic shock who received noradrenaline and hydrocortisone were included. Only patients who exceeded $200 \mathrm{mg} /$ day of hydrocortisone were excluded. The primary outcome was mean blood glucose.

Results: A total of 108 patients (with 3,021 blood glucose readings) were included in the final analysis. Seventy-six patients received hydrocortisone as intermittent boluses (70.3\%), and 32 patients (29.7\%) received continuous infusion. For the primary outcome, no statistically or clinically significant difference was found in the blood glucose estimated marginal mean: $8.58 \mathrm{mmol} / \mathrm{L}$ (95\% confidence interval [Cl]; 8.01-9.16) in the bolus group and $8.9 \mathrm{mmol} / \mathrm{L}(95 \% \mathrm{Cl} ; 7.99-9.82)$ in the infusion group with a mean difference of $0.32 \mathrm{mmol} / \mathrm{L}$ ( $95 \% \mathrm{Cl} ;-0.77$ to 1.41$)$. For secondary outcomes, no difference was found between the two groups in mortality, length of stay, reversal of shock, or hypoglycemic events. Conclusion: Intermittent boluses of hydrocortisone were not associated with a higher risk of hyperglycemia than continuous infusion in septic shock patients.

Keywords: Continuous infusion, Hydrocortisone, Hyperglycemia, Intermittent boluses, Septic shock.

Indian Journal of Critical Care Medicine (2021): 10.5005/jp-journals-10071-23501
\end{abstract}

\section{INTRODUCTION}

Septic shock is a serious condition associated with a high mortality rate, despite advanced diagnostic and therapeutic options. ${ }^{1-3}$ Septic shock is a type of vasodilatory shock clinically identified by the requirement of vasopressors to keep mean arterial blood pressure greater than $65 \mathrm{~mm} \mathrm{Hg}$ and lactate less than $2 \mathrm{mmol} / \mathrm{L}$ in the absence of hypovolemia. ${ }^{4}$

Severe stress conditions may lead to decreased synthesis of cortisol. It has been shown that septic shock patients have lower levels of cortisol. ${ }^{5}$ Corticosteroids may be useful, as they counteract the uncontrolled inflammatory process that characterizes sepsis and restore cardiovascular homeostasis through salt and water retention. ${ }^{6}$ Hydrocortisone showed to be effective in reducing the time until reversal of shock when added to standard therapy. ${ }^{7,8}$

Hyperglycemia is one of the commonly reported adverse side effects of corticosteroid treatment. ${ }^{8,9}$ Cumulative evidence supports that hyperglycemia may be associated with a higher incidence of mortality in critically ill patients. ${ }^{10,11}$ Some studies showed a higher incidence of hyperglycemia with hydrocortisone given as intermittent boluses when compared to continuous infusion. ${ }^{12-14}$ However, these studies were conducted on relatively small sample sizes.

The latest surviving sepsis campaign guidelines did not recommend a specific administration method for hydrocortisone due to the lack of enough evidence to prove the association between intermittent hydrocortisone boluses and hyperglycemia. ${ }^{15}$ The objective of this study was to bridge this gap in the literature by evaluating the risk of hyperglycemia of intermittent boluses vs
${ }^{1-3}$ Pharmacy Department, Al-Wakra Hospital, Hamad Medical Corporation, Al-Wakra, Qatar

${ }^{4}$ Pharmacy Department, Saint Luke's Hospital of Kansas City, Kansas City, Missouri, USA

Corresponding Author: Hassan Mitwally, Pharmacy Department, AlWakra Hospital, Hamad Medical Corporation, Al-Wakra, Qatar, Phone: +97455186273, e-mail: hmitwally@hamad.qa

How to cite this article: Mitwally H, Saad MO, Mahmoud S, Mohamed A. Hyperglycemia Risk Evaluation of Hydrocortisone Intermittent Boluses vs Continuous Infusion in Septic Shock: A Retrospective Study. Indian J Crit Care Med 2021;25(1):29-33.

Source of support: Medical Research Center, Hamad Medical Corporation, Qatar (Internal funding).

Conflict of interest: None

continuous infusion of hydrocortisone on a relatively large sample size of critically ill patients with septic shock.

\section{Materials and Methods}

\section{Patients}

This was a retrospective cohort observational study conducted in two intensive care units (ICUs) (medical and surgical) in Qatar. Data were collected retrospectively from the electronic medical records of eligible patients admitted to ICUs between June 12, 2015, and December 20, 2017. 


\section{Inclusion Criteria}

All patients who were admitted to the medical ICU (MICU) or surgical ICU (SICU) with septic shock were started on norepinephrine (norepinephrine is our first-line vasopressor in treating septic shock) and received hydrocortisone either as intermittent boluses or as continuous infusion.

\section{Exclusion Criteria}

Patients who exceeded $200 \mathrm{mg} /$ day of hydrocortisone.

Data were collected from electronic medical records, including age, sex, history of hypertension, diabetes, coronary artery disease, chronic kidney disease, chronic respiratory disease and cancer, recent use of corticosteroids, source of infection, type of admission (surgical or medical), individual parameters of the sequential organ failure assessment (SOFA) score, hospital length of stay, ICU length of stay, blood glucose readings, and mortality.

\section{Study Outcomes}

The primary outcome was mean blood glucose. Secondary efficacy outcomes were mortality, duration until reversal of shock (defined as the duration of vasopressor therapy), hospital length of stay, and ICU length of stay. Secondary safety outcomes were the incidence of hypoglycemia, hyperglycemia, and glycemic variability estimated as the glucose coefficient of variation (standard deviation/mean $\times 100$ ). All blood glucose values were included in the analysis (either arterial or venous whole blood sampling or fingerstick capillary testing).

\section{Statistical Analysis}

Baseline characteristics were described as mean with standard deviation for continuous variables, median with interquartile range for ordinal variables and non-normally distributed continuous variables, and frequencies with percentages for categorical variables. They were compared using a $t$ test for normal continuous variables, a Wilcoxon rank-sum test for ordinal and non-normal continuous variables, and a Chi-square test for categorical variables.

To account for multiple blood glucose measurements of the glucose levels in each patient, which results in the dependence of observations, glycemic control was assessed using a linear mixed regression model with an unstructured covariance matrix assuming independence between patients. The analysis was adjusted for history of diabetes, median baseline glucose level, and chronic steroid use as fixed effects and the duration of hydrocortisone therapy as a random effect. The estimated marginal means of blood glucose and the mean difference between the two groups were calculated from the linear mixed model. Mortality was compared using multiple logistic regressions with adjustment for baseline age, SOFA score, surgical status, and comorbidities (diabetes, hypertension, chronic kidney disease, cancer, coronary artery disease, and respiratory diseases) with results reported as crude odds ratios and adjusted odds ratios with corresponding $95 \% \mathrm{Cls}$. The incidences of hyperglycemia and hypoglycemia were compared using a Chi-square test, hospital length of stay, ICU length of stay, and vasopressor therapy were compared using a Wilcoxon rank-sum test, and the coefficient of blood glucose variation was compared using an independent $t$ test.

All statistical analyzes were performed using the Statistical Package for Social Sciences (SPSS) program version 22.0 (International Business Machines [IBM] SPSS Statistics for Windows; IBM Corp, Armonk, New York [NY]). $p$ values $<0.05$ were considered significant.

\section{Results}

\section{Baseline Characteristics}

A total of 108 patients (with 3,021 blood glucose readings) were included in the final analysis. Seventy-six (70.3\%) patients received hydrocortisone as intermittent boluses, and 32 (29.7\%) patients received continuous infusion (Table 1). All patients in both groups received a total of $200 \mathrm{mg} /$ day of hydrocortisone. In the continuous infusion group, the patients received $200 \mathrm{mg}$ infused over 24 hours, and in the boluses group, the patients received $50 \mathrm{mg}$ every 6 hours. The mean ages of the infusion and bolus groups were 55 and 64 years, respectively. Other baseline characteristics were similar in both groups except for hypertension, cancer, chronic kidney disease, and the number of patients who received corticosteroids before admission (Table 1). Additionally, more patients received hydrocortisone as intermittent boluses in the MICU (64 patients [84.2\%]), and more patients received hydrocortisone as infusion in the SICU ( $p$ values $<0.001$ ). There was no difference between the two groups in hydrocortisone duration (Table 1).

\section{Outcomes}

For the primary outcome, the blood glucose estimated marginal mean was $8.58 \mathrm{mmol} / \mathrm{L}$ (95\% confidence interval [Cl]; 8.01 to 9.16) for the bolus group and $8.9 \mathrm{mmol} / \mathrm{L}$ ( $95 \% \mathrm{Cl} ; 7.99$ to 9.82 ) for the infusion group with a mean difference of $0.32 \mathrm{mmol} / \mathrm{L}(95 \% \mathrm{Cl} ;-0.77$ to 1.41 ) (Table 2). For the secondary efficacy outcomes, 52 (68\%) of 76 in the bolus group and 16 (50\%) out of 32 in the infusion group died with an adjusted odds ratio of 0.62 (0.19-2.04). No statistically significant difference was observed between the two groups with regard to hospital length of stay, ICU length of stay, or vasopressor duration (Table 3). For the secondary safety outcomes, no statistical significance was found between the two groups in hyperglycemia, hypoglycemia incidence, or glucose coefficient of variation (Table 3).

\section{Discussion}

The objective of this study was to determine the safest administration method of hydrocortisone in septic shock patients. In this study, the administration of hydrocortisone as intermittent boluses caused neither an increase in mean blood glucose nor more hyperglycemic events than continuous infusion. The difference between the blood glucose means was $0.32 \mathrm{mmol} / \mathrm{L}$ more in the bolus group, which is not a statistically or clinically significant difference. There were no significant differences between the two groups regarding the secondary efficacy of safety outcomes. Most of the SICU patients received hydrocortisone as infusion, and most of the MICU patients received intermittent boluses of hydrocortisone. More patients overall received hydrocortisone as intermittent boluses than continuous infusion, however, because the MICU admission rate in our facility is higher than that of the SICU. This discrepancy in practice was one of the triggers to conduct this study.

In a small randomized control trial, Weber-Carstens et al. found that hydrocortisone was associated with a significant increase in blood glucose readings within 6 hours after bolus doses when compared to continuous infusion. ${ }^{12}$ This study was conducted on only 16 patients, and the authors did not report baseline characteristics. Another randomized control trial conducted on 48 patients by Loisa et al. reported that bolus doses of hydrocortisone increased the risk of hyperglycemia. ${ }^{13}$ Despite being statistically significant, the results were not clinically significant, as the difference in mean blood glucose was only $0.2 \mathrm{mmol} / \mathrm{L}$. Moreover, 
Table 1: Baseline characteristics of patients

\begin{tabular}{|c|c|c|c|c|}
\hline & $\begin{array}{l}\text { Bolus group } \\
(n=76)\end{array}$ & $\begin{array}{l}\text { Infusion group } \\
(n=32)\end{array}$ & p value & $\begin{array}{l}\text { Overall } \\
(n=108)\end{array}$ \\
\hline Age-years, mean (SD) & $64(24)$ & $55(33.8)$ & 0.024 & $63(28.3)$ \\
\hline Male sex, $n(\%)$ & $47(61.8)$ & $23(71.9)$ & 0.319 & $70(64.8)$ \\
\hline Diabetes mellitus, $n$ (\%) & $41(53.9)$ & $12(37.5)$ & 0.118 & $53(49.1)$ \\
\hline Hypertension, $n(\%)$ & $52(68.4)$ & $13(40.6)$ & 0.007 & $65(60.2)$ \\
\hline Cancer, $n(\%)$ & $17(22.4)$ & $2(6.3)$ & 0.045 & $19(17.6)$ \\
\hline Coronary artery disease, $n(\%)$ & $33(43.4)$ & $12(37.5)$ & 0.569 & $45(41.7)$ \\
\hline Chronic kidney disease, $n$ (\%) & $36(47.4)$ & $4(12.5)$ & 0.001 & $40(37)$ \\
\hline Respiratory disorder, $n(\%)$ & $14(18.4)$ & $5(15.6)$ & 0.727 & $19(17.6)$ \\
\hline On steroids before admission, $n(\%)$ & $9(11.6)$ & $0(0)$ & 0.036 & $9(8.3)$ \\
\hline Intensive care unit, $n$ (\%) & & & $<0.001$ & \\
\hline Medical & $64(84.2)$ & $16(50)$ & & $80(74.1)$ \\
\hline Surgical & $12(15.8)$ & $16(50)$ & & $28(25.9)$ \\
\hline Source of infection, $n$ (\%) & & & 0.374 & \\
\hline Lung & $28(36.8)$ & $9(28.1)$ & & $37(34.3)$ \\
\hline Abdomen & $6(7.9)$ & $6(18.8)$ & & $12(11.1)$ \\
\hline Urinary tract & $14(18.4)$ & $3(9.4)$ & & $17(15.7)$ \\
\hline Central line-associated & $1(1.3)$ & $1(3.1)$ & & $2(1.9)$ \\
\hline Skin and soft tissue & $8(10.5)$ & $5(15.6)$ & & $13(12)$ \\
\hline Other & $19(25)$ & $8(25)$ & & $27(25)$ \\
\hline Etomidate use for intubation, $n(\%)$ & $17(22.4)$ & $6(18.8)$ & 0.675 & $23(21.3)$ \\
\hline SOFA score, median (IQR) & $12(5)$ & $14(5)$ & 0.062 & $13(4)$ \\
\hline $\mathrm{PaO}_{2} / \mathrm{FiO}_{2}$ ratio, median (IQR) & $224(208)$ & $178(186)$ & 0.113 & $217(208)$ \\
\hline Platelets count $-10^{3} / \mu \mathrm{L}$, median (IQR) & $120(140)$ & 97 (109) & 0.08 & 109 (129.3) \\
\hline Glasgow coma scale, median (IQR) & $8(11)$ & $6.5(5)$ & 0.08 & $7(7)$ \\
\hline Bilirubin- $\mu \mathrm{mol} / \mathrm{L}$, median (IQR) & $27(46.8)$ & $30.5(43.5)$ & 0.742 & $29(46.5)$ \\
\hline Creatinine- $\mu \mathrm{mol} / \mathrm{L}$, median (IQR) & $232(185)$ & $198.5(150.5)$ & 0.321 & $211(165)$ \\
\hline Hydrocortisone duration-days, mean (SD) & 2.46 & 2.44 & 0.97 & - \\
\hline
\end{tabular}

IQR, interquartile range; $\mathrm{FiO}_{2}$, fraction of inspired oxygen; $\mathrm{PaO}_{2}$, partial pressure arterial oxygen; $\mathrm{SD}$, standard deviation; SOFA, sequential organ failure assessment

Table 2: Primary outcome

\begin{tabular}{|c|c|c|c|}
\hline & Bolus $(n=76)$ & Infusion $(n=31)^{a}$ & $\begin{array}{l}\text { Mean difference, } 95 \% \\
\text { confidence interval }\end{array}$ \\
\hline $\begin{array}{l}\text { Blood glucose }(\mathrm{mmol} / \mathrm{L}) \text {, estimated } \\
\text { marginal mean }\end{array}$ & $8.58(8.01-9.16)$ & $8.9(7.99-9.82)$ & $0.32(-0.77-1.41)^{b}$ \\
\hline \multicolumn{4}{|c|}{$\begin{array}{l}\text { aBlood glucose readings were not available for one patient } \\
{ }^{\mathrm{b}} \text { Adjusted for diabetes, median baseline glucose, duration of hydrocortisone therapy and previous chronic steroi } \\
\text { use (using linear mixed model with an unstructured covariance matrix, assuming independence across patient } \\
\text { with diabetes history, median baseline glucose and chronic steroid use as fixed effects and duration of hydrocort } \\
\text { sone therapy as random effect) }\end{array}$} \\
\hline
\end{tabular}

the baseline comorbidities of the two groups were not mentioned. Another notable point in Loisa et al.'s study is that they used a cutoff of $7 \mathrm{mmol} / \mathrm{L}$ for defining hyperglycemia, although the last surviving sepsis guidelines accept up to $10 \mathrm{mmol} / \mathrm{L} .{ }^{13}$ A retrospective cohort study by Hoang et al. reported a higher incidence of hyperglycemia with intermittent bolus doses of hydrocortisone. ${ }^{14}$ However, this study was conducted on only 51 patients, and the results were not adjusted for confounders, such as diabetes. They also noted a higher prevalence of diabetic patients in the bolus group, which may have affected the results.

In contrast, a recent randomized control trial conducted on 29 patients by Tilouche et al. found no difference between bolus and infusion; however, the sample size was small, and hyperglycemia was not the primary outcome. ${ }^{16}$ Gibbison et al. conducted a metaanalysis comparing different regimes of steroids in septic shock patients including hydrocortisone bolus and infusion, although they did not assess the difference in hyperglycemia between different regimens due to significant variation in defining hyperglycemia between trials.

This study reports no difference in mortality between the two groups. These findings correlate with previous studies comparing boluses and continuous infusion..$^{13,14,16}$ Tilouche et al. found that hydrocortisone boluses aided faster reversal of shock than continuous infusion, ${ }^{16}$ but their study showed no significant 
Hyperglycemia Risk of Hydrocortisone Boluses vs Infusion in Septic Shock

Table 3: Secondary outcomes

\begin{tabular}{|c|c|c|c|c|}
\hline Secondary efficacy outcome & Bolus $(n=76)$ & Infusion $(n=32)$ & Odds ratio (OR) & Adjusted OR $(\mathrm{Cl})^{a}$ \\
\hline Mortality, $n(\%)$ & $52(68.4)$ & $16(50)$ & $0.46(0.2-1.07)$ & $0.62(0.19-2.04)$ \\
\hline Other secondary efficacy outcomes ${ }^{b}$ & Bolus $(n=24)$ & Infusion $(n=16)$ & $p$ value ${ }^{c}$ & \\
\hline ICU stay (days), median (IQR) & $9.5(14)$ & $12(17)$ & 0.58 & \\
\hline Hospital stay (days), median (IQR) & $22(61)$ & $19.5(70.8)$ & 0.99 & \\
\hline Vasopressor therapy (days), median (IQR) & $1.7(1.9)$ & $2.3(1.3)$ & 0.1 & \\
\hline Secondary safety outcomes & Bolus $(n=76)$ & Infusion $(n=31)^{d}$ & pvalue & \\
\hline Patients with any hyperglycemia ${ }^{\mathrm{e}}, n(\%)$ & $51(67.1)$ & $22(71)$ & $0.7^{\mathrm{g}}$ & \\
\hline Patients with any hypoglycemia ${ }^{f}, n(\%)$ & $33(43.4)$ & $13(41.9)$ & $0.89^{9}$ & \\
\hline Glucose coefficient of variation, mean (SD) & $32.2(20.3)$ & $30.5(17.9)$ & $0.69^{h}$ & \\
\hline Insulin requirements-units/day, median (IQR) & $0(11.5)$ & $0(11.5)$ & $0.628^{c}$ & \\
\hline
\end{tabular}

$\mathrm{Cl}$, confidence interval; ICU, intensive care unit; IQR, interquartile range; SD, standard deviation

${ }^{a}$ Adjusted for age, SOFA score, surgical status and comorbidities (diabetes, hypertension, chronic kidney disease, cancer, coronary artery disease and respiratory diseases) using multiple logistic regression, the reference group is the bolus

${ }^{b}$ Calculated among survivors only

'Using Wilcoxon rank sum test

${ }^{\mathrm{d}}$ Blood glucose readings were not available for one patient

eHyperglycemia; defined as blood glucose reading $>10 \mathrm{mmol} / \mathrm{L}$

fHypoglycemia; defined as blood glucose reading $<4 \mathrm{mmol} / \mathrm{L}$

IUsing Chi-squared test

hUsing $t$-test

difference in the times until shock reversal between the two groups. Although they did discover a trend toward decreased vasopressor duration in the bolus group by a median of 0.6 days, it was not statistically significant (Table 3 ).

The strengths of the present study include its relatively larger sample size than previous studies addressing the same clinical question and the adjustment of the results to baseline characteristics (Table 2), as many patient factors other than hydrocortisone may affect blood glucose readings. The chief limitation, however, is the study's design. Being retrospective rather than prospective might have introduced some bias to the results. However, the objective nature of the outcomes and the use of electronic medical records may have decreased the risk of bias. Another limitation was the use of multiple methods of blood glucose measurement (laboratory, blood gases, and point of care). We acknowledge that the corresponding readings from these methods may slightly differ. However, we believe that including a substantially large number of blood glucose values from different methods outweighs this limitation.

These findings will facilitate the administration of hydrocortisone among different facilities, as practitioners will not require changing their administration methods. Additionally, some clinicians may prefer to give hydrocortisone as boluses based on the studies, which showed that boluses may cause an early reversal of shock and also ease administration more than continuous infusion.

\section{CONCLUSION}

In septic shock patients, hydrocortisone given as intermittent boluses was not associated with higher risk of hyperglycemia than continuous infusion. Larger prospective studies are required to confirm these results.

\section{ACKnowledgments}

We thank Amy Ann Mathew, RPh and Peter Adel, RPh for their assistance with data collection.

\section{Ethical Approval}

This study was approved by the ethical committee, Medical research center, Hamad Medical Corporation, under number: MRC-01-18-129.

\section{References}

1. Angus DC, Linde-Zwirble WT, Lidicker J, Clermont G, Carcillo J, Pinsky MR, et al. Epidemiology of severe sepsis in the United States: analysis of incidence, outcome, and associated costs of care. Crit Care Med 2001;29(7):1303-1310. DOI: 10.1097/00003246-200107000-00002.

2. Zhou J, Qian C, Zhao M, Yu X, Kang Y, Ma X, et al. Epidemiology and outcome of severe sepsis and septic shock in intensive care units in mainland China. PLoS ONE 2014;9(9):e107181. DOI: https://doi. org/10.1371/journal.pone.0107181.

3. McPherson D, Griffiths C, Williams M, Baker A, Klodawski E, Jacobson $B$, et al. Sepsis-associated mortality in England: an analysis of multiple cause of death data from 2001 to 2010. BMJ Open 2013;3(8):e002586. DOI: https://doi.org/10.1136/bmjopen-2013-002586.

4. Singer M, Deutschman CS, Seymour CW, Shankar-Hari M, Annane $D$, Bauer $\mathrm{M}$, et al. The third international consensus definitions for sepsis and septic shock (sepsis-3). JAMA 2016;315(8):801. DOI: 10.1001/ jama.2016.0287.

5. Annane D. Adrenal insufficiency in sepsis. Curr Pharm Des 2008;14(19):1882-1886. DOI: 10.2174/138161208784980626.

6. Annane D. Corticosteroids for severe sepsis: an evidence-based guide for physicians. Ann Intensive Care 2011;1(1):7. DOI: 10.1186/21105820-1-7.

7. Gibbison B, López-López JA, Higgins JPT, Miller T, Angelini GD, Lightman SL, et al. Corticosteroids in septic shock: a systematic review and network meta-analysis. Crit Care 2017;21(1):78. DOI: 10.1186/ s13054-017-1659-4.

8. Venkatesh B, Finfer S, Cohen J, Rajbhandari D, Arabi Y, Bellomo R, et al. Adjunctive glucocorticoid therapy in patients with septic shock. N Engl J Med 2018;378(9):797-808. DOI: 10.1056/NEJMoa1705835.

9. Sprung CL, Annane D, Keh D, Moreno R, Singer M, Freivogel K, et al. Hydrocortisone therapy for patients with septic shock. N Engl J Med 2008;358(2):111-124. DOI: 10.1056/NEJMoa071366

10. Krinsley JS. Association between hyperglycemia and increased hospital mortality in a heterogeneous population of critically ill patients. Mayo Clin Proc 2003;78(12):1471-1478. DOI: https://doi. org/10.4065/78.12.1471 
11. Falciglia M, Freyberg RW, Almenoff PL, D'Alessio DA, Render ML. Hyperglycemia-related mortality in critically ill patients varies with admission diagnosis. Crit Care Med 2009;37(12):3001-3009. DOI: 10.1097/CCM.0b013e3181b083f7.

12. Weber-Carstens S, Deja M, Bercker S, Dimroth A, Ahlers O, Kaisers $\mathrm{U}$, et al. Impact of bolus application of low-dose hydrocortisone on glycemic control in septic shock patients. Intensive Care Med 2007;33(4):730-733. DOI: 10.1007/s00134-007-0540-3.

13. Loisa P, Parviainen I, Tenhunen J, Hovilehto S, Ruokonen E. Effect of mode of hydrocortisone administration on glycemic control in patients with septic shock: a prospective randomized trial. Crit Care 2007;11(1):R21. DOI: 10.1186/cc5696.
14. Hoang $H$, Wang S, Islam S, Hanna A, Axelrad A, Brathwaite C. Evaluation of hydrocortisone continuous infusion versus intermittent boluses in resolution of septic shock. P T 2017;42(4): 252-255.

15. Rhodes A, Evans LE, Alhazzani W, Levy MM, Antonelli M, Ferrer $R$, et al. Surviving sepsis campaign: international guidelines for management of sepsis and septic shock: 2016. Intensive Care Med 2017;43(3):304-377. DOI: 10.1007/s00134-017-4683-6.

16. Tilouche N, Jaoued O, Ali HBS, Gharbi R, Hassen MF, Elatrous S. Comparison between continuous and intermittent administration of hydrocortisone during septic shock. Shock 2019;52(5):481-486. DOI: $10.1097 /$ SHK.0000000000001316. 